\title{
A Precise Fast High Voltage Pulse Measurement Optical System Using Kerr Cell Containing Nitrobenzene as an Optically Active Material
}

\author{
Babar Hussain, Masroor Ikram, and Asif Mehmood
}

\begin{abstract}
High voltage pulses are much more difficult to measure precisely because of sensitivity of electrical devices to electromagnetic noise. One commonly used technique to measure high voltage pulses, is the use of Kerr cell containing any optically active material (nitrobenzene in our case). This work is based on optical technique to measure high voltage fast pulses precisely. Optical characteristics of nitrobenzene are exploited to measure the pulses. We have proposed a substitute design of Kerr cell to prevent breakdown in nitrobenzene which causes severe problems in measurement. Kerr cells of different sizes and different types are used in the experiments to measure the high voltage pulses of peak amplitude up to $100 \mathrm{kV}$.
\end{abstract}

Index Terms-Kerr cell, high voltage, pulse measurement, nitrobenzene, birefringence.

\section{INTRODUCTION}

A recent increase in electric power demand causes generation of high voltage pulses, which in turn requires improving the measurement and preventive methods for the power apparatus. Considerable electromagnetic noise may be generated, rising the need of a nearly perfect measurement system which is immune to electromagnetic noise. For this purpose, it is highly necessary to develop better measuring techniques for the fast transient impulse voltages. To overcome the above stated problems an electro-optic Kerr effect is used for accurate measurements of fast rising (of order $10^{-8} \mathrm{~s}$ ) high voltage pulses [1]-[3]. This system can be very small and portable. It provides nearly perfect electrical isolation between the much high voltage circuit and sensitive measuring instruments. Optical measurement system shows high signal to noise ratio (SNR) by separating electrically the measuring system from noise sources of the power system [4], [5]. Objective of this paper is to show the work related to design and implementation of a precise fast high voltage pulse measurement system using electro-optical techniques. Main component of this system is a glass tube containing optically active material called Kerr Cell. Nitrobenzene in liquid form is used as an optically active material.

Optical techniques for the measurement of current or voltage at high amplitude levels can be divided into two

Manuscript received March 9, 2013; revised April 25, 2013.

Babar Hussain is with Ph.D. student at Department of ECE, University of North Carolina Charlotte, USA (e-mail: bhussai1 @uncc.edu).

Masroor Ikram is with Head Department of Physics and Applied Mathematics, Pakistan Institute of Engineering and Applied Sciences, Islamabad, Pakistan (e-mail: masroor@pieas.edu.pk).

Asif Mehmood is with Senior Scientist, Department of Technical Development, Islamabd, Pakistan (e-mail: asif3172@yahoo.com). general groups. The first includes measurement systems which exploit the interaction of an electric or magnetic field with a material to cause a change in the index of refraction [6]. These systems are based on the Faraday, Kerr, or Pockels effect. The second approach uses a light source, usually a semiconductor laser or a light-emitting diode, located on the high voltage line to transmit information concerning voltage, current, or power to a lower voltage level [7], [8]. There has been substantial development using this approach of both laboratory instruments and commercial prototypes. Former approach is used in this work to measure high voltage pulses.

\section{THEORY OF KERR CELl OPERATION}

Fig. 1 shows a typical setup of Kerr cell system for high voltage measurement. Kerr cell is an electro-optic transducer whose optical characteristics are highly dependent on applied electric field. In the absence of an applied electric field no significance change occurs in the orientation of plane of polarization of light. But when an electric field is applied across the Kerr medium (nitrobenzene in our case) by applying voltage across the Kerr cell electrodes, birefringence occurs in the medium and due to different refractive indices offered by birefringent material, a delay occurs in the two components of electric field of light, which is at an angle of $-45^{\circ}$ with the applied electric field, and light which is initially linearly polarized, becomes elliptically polarized [9]. This change can be assumed as rotation of plane of polarization of light and can be sensed by a photodetector circuit after passing it through an analyzer. Light should be passed through a polarizer before entering the Kerr cell, whose optical axis is at $-45^{\circ}$ to the applied electric field [10], [11]. If polarizer and analyzer are perpendicular to each other then negligible light intensity is detected at the output of analyzer by the photodetector circuit.

Light intensity is detected by the photodetector circuit at the output of the analyzer. If we keep on increasing the applied voltage across the electrodes of the Kerr cell, output of photodetector reaches its peak value which corresponds to a certain value of applied voltage. This variation in output transmittance of light is due to the difference in refractive indices offered by nitrobenzene to the two components of electric field of light. This difference is given by [6]

$$
\Delta n=\lambda \mathrm{KE}^{2}
$$

where $\Delta n$ is the change in refractive indices offered by electro-optical birefringent material to the two perpendicular components of light, $\lambda$ is wavelength of light passing 
through medium, $K$ is the Kerr constant of birefringent material and $E$ is applied electric field. Corresponding phase difference in the components can be found as

$$
\Delta \Phi=(2 \pi \lambda) L \Delta n
$$

where $L$ is the length of each Kerr cell electrode. Using (1) we get

$$
\Delta \Phi=2 \pi K L E^{2}
$$

Or

$$
\Delta \Phi=\frac{2 \pi K L V^{2}}{d^{2}}
$$

which is the final relation between applied voltage across the electrodes of Kerr cell and corresponding rotation in polarization plane of light. This rotation in polarization plane can be calculated by Malus' law which is mathematically defined as

$$
I=I_{0} \cos ^{2} \Phi
$$

where $I$ is the instantaneous intensity of light, $I_{0}$ is the maximum intensity of light and $\Phi$ is the instantaneous angle between plane of polarization of light and optical axis of analyzer.

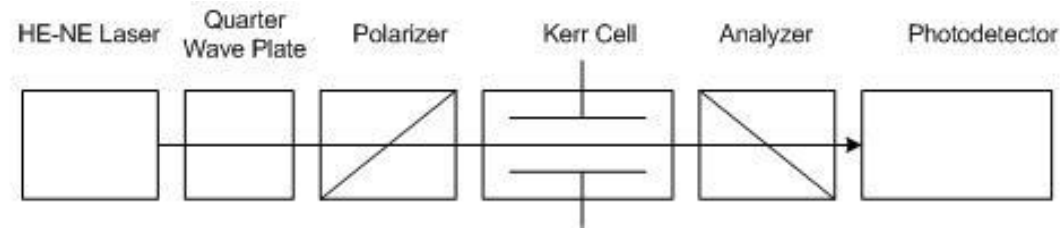

Fig. 1. Block diagram of the experimental setup.

\section{EXPERIMENTAL SETUP}

Fig. 2 shows different types of Kerr cells used in our experiments. Size of Kerr cell depends on the amplitude of pulse we want to measure. Mainly two types of Kerr cells were used. One type contains nitrobenzene having direct contact with electrodes therefore more probability of conduction through nitrobenzene. In second type Kerr cell is constructed in such a way that nitrobenzene is isolated from electrodes by a thin glass sheet which prevents flow of large current through nitrobenzene.

In this approach formula which relates voltage and electric field is needed to be modified as [8]

$$
E=\frac{V}{d+\frac{2 \varepsilon d_{g}}{\varepsilon_{g}}}
$$
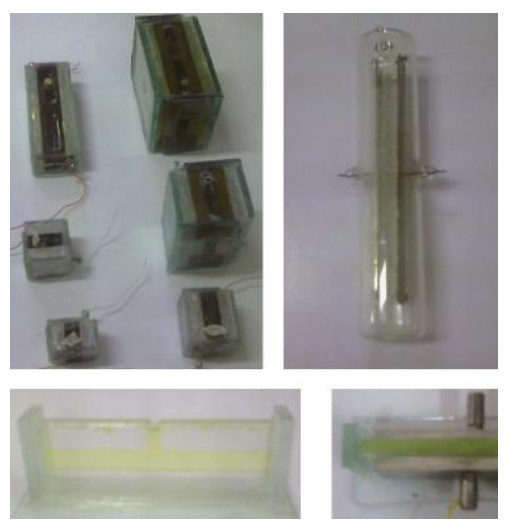

Fig. 2. Different Kerr cells used in experiments

where $d$ is the width of Kerr medium (nitrobenzene), $\mathcal{E}$ is dielectric strength of Kerr medium, $d_{g}$ is the width of glass which separates nitrobenzene and electrodes and $\varepsilon_{g}$ is dielectric strength of glass. Fig. 3 shows this arrangement.

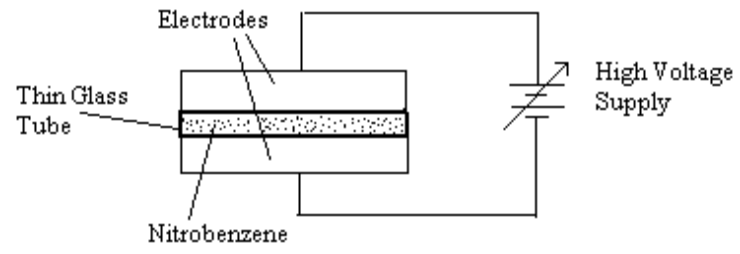

Fig. 3. Kerr cell with isolated nitrobenzene

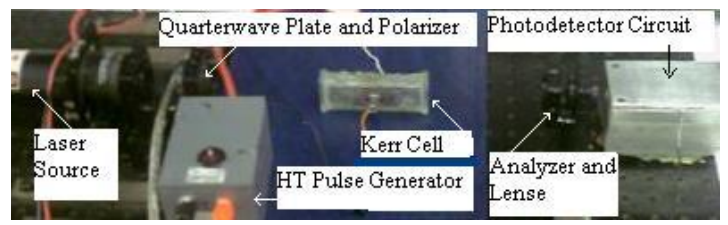

Fig. 4. Complete experimental setup

Fig. 4 shows arrangement of completely aligned experimental setup for high voltage pulse measurement using Kerr cell. Kerr cell in this setup is one in which electrodes have no direct contact with nitrobenzene. Therefore electric field in nitrobenzene in this setup is not the ratio of applied voltage to the separation between electrodes but is given by (6) because we have to take into account the dielectric strength as well as the thickness of both mediums (nitrobenzene and glass) between the electrodes.

\section{RESULTS AND DISCUSSION}

A simulation result of $12.5 \mathrm{kV}$ pulse is shown in Fig. 5 . In this simulation length of electrodes of Kerr cell is $11.6 \mathrm{~cm}$ and separation between them is $0.35 \mathrm{~cm}$. Kerr constant of nitrobenzene is kept $2.44 \times 10^{-10} / \mathrm{V}^{2}$. If distance between the electrodes is increased up to $0.5 \mathrm{~cm}$ then electric field in nitrobenzene reduces and effectively there will be less 
number of cycles at the output as shown in Fig. 6.

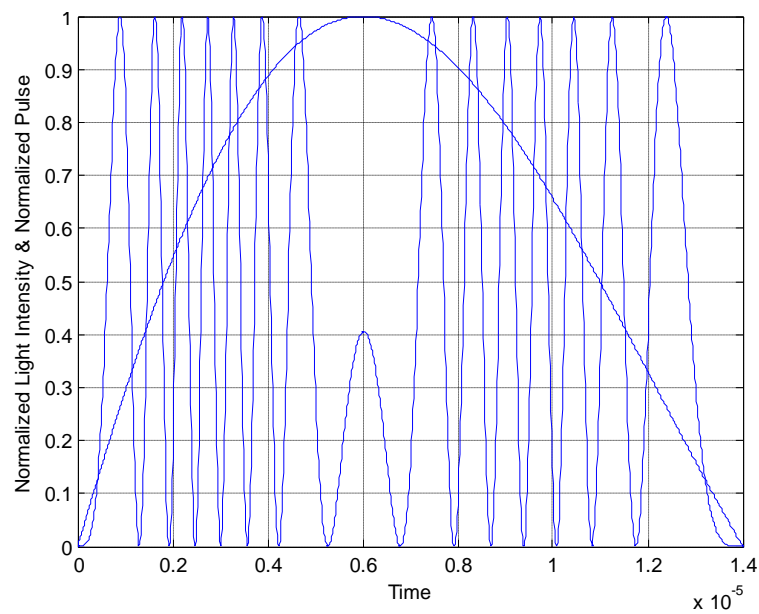

Fig. 5. Simulation of $12.5 \mathrm{kV}$ pulse $(d=0.35 \mathrm{~cm})$

There are two methods mostly adopted for the measurement of high voltage pulse from output data of photodetector. One convenient but less accurate method is to count the output pulses and using (4) as

$$
V_{p}=\sqrt{\frac{N d^{2}}{2 K L}}
$$

where $V_{p}$ is the peak value of pulse to be measured and $N$ is number of pulses before pulse peak occurs. Equation (7) is valid for the arrangement shown in Fig. 1 where there is only nitrobenzene between the electrodes as dielectric. If arrangement shown in Fig. 3 is used where nitrobenzene is isolated from electrodes by glass then (6) and (4) can be used to measure peak value of pulse as

$$
V_{p}=\sqrt{\frac{N}{2 K L}}\left(d+\frac{2 \varepsilon d_{g}}{\varepsilon_{g}}\right)
$$

Another method which is more accurate needs some data acquisition techniques and computer programming in which output data from photodetector is stored in PC for further processing to reconstruct input high voltage pulse. Some outputs obtained at digital oscilloscope are shown in Fig. 7 and Fig. 8. Breakdown occurred in nitrobenzene can be noted in Fig. 8.

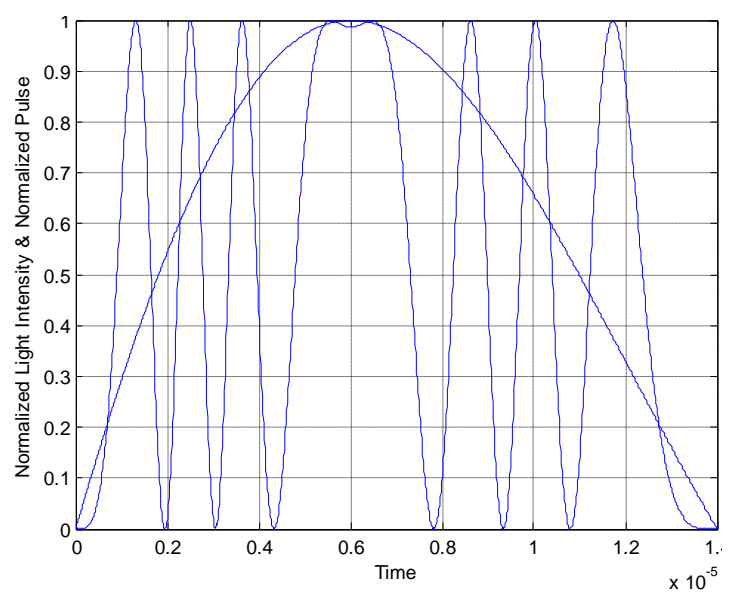

Fig. 6. Simulation of $12.5 \mathrm{kV}$ pulse $(\mathrm{d}=0.5 \mathrm{~cm})$
Fig. 7 shows the result of experimental setup when Kerr cell with isolated nitrobenzene was used and Fig. 8 shows the result when nitrobenzene was in direct contact with electrodes.



Fig. 7. Output waveform for $100 \mathrm{kV}$ pulse

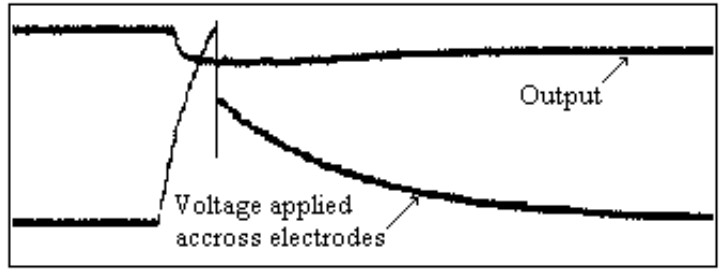

Fig. 8. Output showing breakdown in nitrobenzene

\section{CONCLUSION}

In this paper we have explained Kerr effect and its utilization in measurement of fast high voltage pulses. This optical technique is much affective and fast enough to measure pulses of duration of order 10ns. We have also proposed an alternative method of constructing Kerr cell to prevent breakdown in nitrobenzene which causes severe problems in measurement. Kerr cells of different sizes and different types are used for the experiments to measure the high voltage pulses of peak amplitude up to $100 \mathrm{kV}$. It is also noted that purity of nitrobenzene is an important issue regarding conduction and breakdown in nitrobenzene. We are working on this system to improve its precision and to apply the Kerr cell to other applications like modulation of light and detection of electric transmission line faults.

\section{REFERENCES}

[1] J. D. Cross and R. Tobazeon, "Electric field distortions produced by solid dielectric spacers separating uniform field electrodes in nitrobenzene," IEEE Transactions on Electrical Insulation, vol. 8, no. 1, 1973, pp. 25-29.

[2] H. Ihori, Y. Ishihara, S. Yano, and M. Fujii, "Continuous measurements of electric field vector maps in nitrobenzene and propylene carbonate," in Proc. 2005 International Symposium on Electrical Insulating Materials, Kitakyushu, Japan, 2005, pp. 564567.

[3] A. J. Duerinckx, H. A. Vanherzeele, J. V. Eck, and A. E. Siegman, "Pulse compression inside an Actively AM Mode-Locked ND:YAG laser using a liquid kerr cell," IEEE Journal of Quantum Electronics, 1978 , vol. 14, no. 12, pp. 983-992.

[4] Y. Zhu and T. Takada, "A 2-Dimensional kerr effect technique for electric field distribution in liquid dielectrics," IEEE Transactions on Dielectrics and Electrical Insulation, 1997, vol. 4, no. 6, pp. 748-757.

[5] P. D. Thacher, "Optical effects of fringing fields in kerr cells," IEEE Transactions on Electrical Insulation, 1976, vol. 11, no. 2, pp. 40-50. 
[6] G. J. FitzPatric and E. F. Kelly, "Comparative high voltage impulse measurement," Journal of Research of National Institute of Standards and Technology, vol. 101, no. 5, 1996, pp. 639-658.

[7] R. E. Hebner, R. A. Malewski, and E. C. Cassidy, "Optical methods of electrical measurement at high voltage levels," in Proc. IEEE vol. 65 , no. 11,1977 , pp. $1524-1548$.

[8] T. J. Englert, B. H. Chowdhury, and E. Grigsby, "A laboratory investigation of electro optic kerr effect for detection of electric transmission line faults," IEEE Transactions on Power Delivery, vol. 6, no. 3, 1991, pp. 979-985.

[9] S. Firdous, K. Hassan, and M. Ikram, "Formulation of a mueller matrix for modeling of depolarization and scattering of nitrobenzene in a Kerr cell," Applied Optics, vol. 44, no. 7, 2005, pp. 1171-1177.

[10] A. B. Treshchalov and V. E. Peet, "Spatial-Time dynamics of the discharge pumping and lasing in a $\mathrm{XeCl}$ Excimer Laser," IEEE Journal of Quantum Electronics, vol. 24, no. 2, 1988, pp. 169-176.
[11] A. J. Chenoweth, O. L. Gaddy, and D. F. Holshouser, "Carbon disulfide traveling-wave kerr cells," in Proc. IEEE, vol. 54, no. 10 , 1966, pp. 1414-1418.

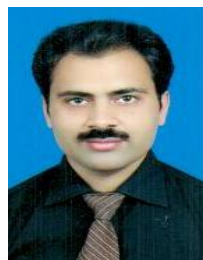

Babar Hussain was born in Mansehra, Pakistan in 1980. He is a Ph.D. student at ECE, UNCC, USA. He research Interests are Optoelectronics, Semiconductor Nanostructures, Femtosecond and Terahertz Pulses. 